\title{
Aggregate consumption behavior and the permanent income hypothesis
}

\section{J. Michael Orszag*}

Department of Economics, University of Michigan, Ann Arbor, MI 48109, USA

\section{Ilya Staroselsky}

Program in Applied and Computational Mathematics, Princeton University, Princeton. NJ 08544, USA

Received 26 October 1992

Accepted 22 December 1992

\begin{abstract}
This paper shows that the random walk behavior of real aggregate consumption is unrelated to Hall's rational choice model. The result implies that random walk behavior of macroeconomic aggregates is something quite general which need have little relation to the optimizing behavior of individual agents.
\end{abstract}

In macroeconomics it has been helpful to consider statistical macroeconomic behavior as close to that of a representative agent model in which the statistical behavior is consistent with maximizing behavior. One must be careful in interpreting the aggregate implications of such models and models of rational behavior would not seem to be directly testable against aggregate data. As an example of these considerations let us consider the model of Hall (1978).

Agents in the economy are characterized by a vector of characteristics $x$ and their consumption at time $t$ is given by $g(x, t)$. There is a distribution $f(x, t)$ of types of consumers in the market. We define $\psi(x, t)=(g(x, t)-\mu(x, t)) f(x, t)$, where $\mu(x, t)$ is the trend level of consumption. We define the deviation of aggregate consumption from trend as $Y(t)$ :

$$
Y(t)=\int \psi(x, t) \mathrm{d} x
$$

Here we will demonstrate that random walk behavior of macroeconomic aggregates is something quite general and can be produced by a wide variety of rather complicated behavior at the microeconomic level. We first consider Hall's model.

$$
\frac{\partial \psi}{\partial t}=\epsilon(x, t),
$$

where $\epsilon$ is noise. For consistency with Hall's approach we also have:

$$
\left\langle\epsilon(x, t) \epsilon\left(x^{\prime}, t^{\prime}\right)\right\rangle=2 M \delta\left(t-t^{\prime}\right) D\left(x, x^{\prime}\right),
$$

* Corresponding author. 
where $M$ is a constant and $D\left(x, x^{\prime}\right)$ is a function which characterizes the correlations of changes in consumption in the space of characteristics. The brackets represent an expectation valuc. The correlation function must be separable in characteristics and time as Hall's theory suggests that noise is delta-correlated in time. We first show that an implication of Hall's theory is indeed that consumption follows a continuous time random walk with drift.

Using the formula

$$
\psi(x, t)=\int L\left(x, x^{\prime}, t-t^{\prime}\right) \epsilon\left(x^{\prime}, t^{\prime}\right) \mathrm{d} x^{\prime} \mathrm{d} t^{\prime},
$$

we obtain the correlation function of micro-level consumption of different types of individuals:

$$
\left\langle\psi\left(x^{\prime}, t^{\prime}\right) \psi(x, t)\right\rangle=C\left(x, x^{\prime}, t-t^{\prime}\right) .
$$

It follows from Eq. (2) that

$$
\begin{aligned}
\frac{\partial C}{\partial t} & -\left\langle\psi(x, t) \epsilon\left(x^{\prime}, t^{\prime}\right)\right\rangle \\
& =M D\left(x, x^{\prime}\right) .
\end{aligned}
$$

We also know that the correlation function of aggregate consumption, $Y(t)$, is

$$
G\left(t-t^{\prime}\right)=\operatorname{cov}\left(Y(t) Y\left(t^{\prime}\right)\right)-\int C\left(x, x^{\prime}, t-t^{\prime}\right) \mathrm{d}^{d} x \mathrm{~d}^{d} x^{\prime},
$$

so that using the above results we have that

$$
\frac{\mathrm{d} G}{\mathrm{~d} t}=N \Rightarrow G \sim N t \Rightarrow \hat{G} \sim \omega^{-2},
$$

where the caret (C) represents a Fourier transform. Therefore, the spectrum of aggregate consumption is the same as that of a continuous time random walk.

The above result does not mean that aggregate random walk behavior is necessarily a consequence of consumer behavior according to the rational choice model. To see this let us consider the following extension of the model (2):

$$
\frac{\partial \psi}{\partial t}=\frac{\partial}{\partial x} \mu(x, t) \frac{\partial \psi}{\partial x}+\frac{\partial}{\partial x} \beta(x, t) \psi+\epsilon(x, t) .
$$

The first term we added represents non-homogeneous diffusion of tastes across consumers and the sccond term represents dependence of the rate of change of consumption on the gradient in characteristic space. We now proceed to show that the correlation function for the aggregate data associated with the above general equation is also a continuous time random walk.

The spatially inhomogeneous equation, Eq. (9), has the following formal solution:

$$
\psi(x, t)=\int L\left(x, x^{\prime}, t-t^{\prime}\right) \epsilon\left(x^{\prime}, t^{\prime}\right) \mathrm{d} x^{\prime} \mathrm{d} t^{\prime},
$$

where $L$ is the Green's function of Eq. (9), i.e. the operator inverse to the semigroup operator on right-hand side of Eq. (9).

It follows from the form of the correlation function that 


$$
\begin{aligned}
\frac{\partial C}{\partial t} & =\frac{\partial}{\partial x} \mu(x, t) \frac{\partial C}{\partial x}+\frac{\partial}{\partial x} \beta(x, t) C+\left\langle\psi(x, t) \epsilon\left(x^{\prime}, t^{\prime}\right)\right\rangle \\
& =\frac{\partial}{\partial x} \mu(x, t) \frac{\partial C}{\partial x}+\frac{\partial}{\partial x} \beta(x, t) C+L\left(x, x^{\prime}, 0\right) M D\left(x, x^{\prime}\right)
\end{aligned}
$$

We also know that the correlation function of aggregate consumption, $Y(t)$, is

$$
G\left(t-t^{\prime}\right)=\operatorname{cov}\left(Y(t) Y\left(t^{\prime}\right)\right)=\int C\left(x, x^{\prime}, t-t^{\prime}\right) \mathrm{d}^{d} x \mathrm{~d}^{d} x^{\prime},
$$

so that it follows that

$$
\frac{\mathrm{d} G}{\mathrm{~d} t}=N \Rightarrow G \sim N t \Rightarrow \hat{G} \sim \omega^{-2}
$$

which is exactly the same spectrum for aggregate behavior as in the rational choice model. This suggests that micro-level behavior can be quite inconsistent with the rational choice model and the aggregates will still behave as a continuous time random walk. Let us consider the implications of the more general equation; all consumers could be arbitrarily far away from satisfying their first-order conditions or consumption could depend on the diffusion of tastes across characteristics and still be completely consistent with a random walk for the aggregate.

The primary objection on economic grounds to our approach is the separability of time and characteristics of the correlation function, but this assumption was exactly what Hall used in deriving his theory. One claim that the rational expectations school as well as the 'New Keynesian' school has made is that it is explicit about its assumptions, but judging from the results here they have been hardly more explicit about macroeconomic structure and market interactions than the old Keynesian school was about the microeconomic structure underlying the models. In conclusion, the permanent income hypothesis is an entirely microeconomic proposition which may or may not be true but which is somewhat unrelated to the study of the dynamics of aggregate consumption behavior.

\section{Reference}

Hall, R., 1978, Stochastic implications of the life cycle-permanent income hypothesis: Theory and evidence, Journal of Political Economy 86, no. 6, 171-186. 